\title{
Sistema Vestible para la Detección Simultánea y No Invasiva del ECG y el Flujo Sanguíneo
}

\section{Wearable System for Simultaneous and Noninvasive Detection of ECG and Blood Flow}

\author{
O. A. Méndez-Lira, A. Gutiérrez-Chávez, J. D. Cota-Ruiz, J. D. Díaz-Román, E. Sifuentes-De la Hoya, R. E. González-Landaeta
}

Universidad Autónoma de Ciudad Juárez

\section{RESUMEN}

Se desarrolló un sistema vestible para la detección simultánea y no invasiva de dos señales provenientes de la actividad cardiaca: el ECG y el flujo sanguíneo. Se utilizaron dos electrodos secos para detectar la derivación I del ECG. La detección del flujo sanguíneo se realizó de forma no invasiva mediante un sensor magnetorresistivo tipo Túnel (TMR) basándose en la firma magnética de la sangre sobre un campo magnético constante proveniente de un imán permanente. Los electrodos, el sensor TMR y el imán se montaron en un brazalete tipo pulsera para la comodidad del usuario. La instrumentación desarrollada para detectar el ECG y el flujo sanguíneo se diseñó en base a una configuración diferencial con una relación de rechazo en modo común superior a 87 dB a 60 Hz y con una tensión de ruido de tal forma que las señales cardiacas presentaron una relación señal a ruido superior a $41 \mathrm{~dB}$, siempre y cuando el sujeto no se mueva durante la medida. La calidad de las señales fue suficiente como para extraer información de dichas señales mediante algoritmos sencillos de implementar. El sistema propuesto es una alternativa a los sistemas vestibles presentados hasta ahora, del cual es posible valorar la salud cardiovascular del sujeto en ambientes no hospitalarios.

PALABRAS CLAVE: sistema vestible; ECG; electrodos secos; flujo sanguíneo; sensor magnetorresistivo 


\begin{abstract}
A wearable system was developed for the simultaneous and non-invasive detection of two signals from cardiac activity: the ECG and blood flow. Two dry electrodes were used to detect the lead I of the ECG. The detection of blood flow was performed non-invasively using a tunnel-magnetoresistance (TMR) sensor based on the magnetic signature of the blood over a constant magnetic field from a permanent magnet. The electrodes, the TMR sensor and the magnet were mounted on a wristband-type bracelet for the user comfort. The instrumentation developed to detect the ECG and blood flow was designed based on a differential configuration with a common-mode rejection ratio higher than $87 \mathrm{~dB}$ at $60 \mathrm{~Hz}$ and with a noise voltage in such a way that the cardiac signals presented a signal-to-noise ratio greater than $41 \mathrm{~dB}$, as long as the subject stays still during the measurement. The quality of the signals was enough to extract information from these signals by means of simple to implement algorithms. The proposed system is an alternative to the wearable systems presented so far, from which it is possible to assess the cardiovascular health of the subject in non-hospital environments.
\end{abstract}

KEYWORDS: wearable system; ECG; dry electrodes; blood flow; magnetoresistive sensor

\section{Correspondencia}

DESTINATARIO: Rafael Eliecer González Landaeta INSTITUCIÓN: Universidad Autónoma de Ciudad Juárez DIRECCIÓN: Av. Plutarco Elías Calles \#1210,

Col. Fovissste Chamizal, C. P. 32310, Ciudad Juárez, Chihuahua, México

CORREO ELECTRÓNICO: rafael.gonzalez@uacj.mx

\section{Fecha de recepción:}

29 de junio de 2018

Fecha de aceptación:

16 de agosto de 2018 


\section{INTRODUCCIÓN}

En los últimos años el monitoreo continuo del estado de salud de los individuos se ha convertido en un tema de gran interés para la comunidad científica e industrial ${ }^{[1]}$. Se ha demostrado que durante las actividades cotidianas que realiza una persona, se pueden presentar eventos atípicos que no se manifiestan en un centro de salud, donde la monitorización de los parámetros fisiológicos se realiza en unas condiciones controladas. Es por eso que se han hecho esfuerzos en desarrollar sistemas portables, incorporados en prendas de vestir (vestibles) y en enseres de uso doméstico o de oficina. Estos sistemas supervisan continua o periódicamente el estado de salud de una persona mientras realiza sus actividades cotidianas durante semanas e incluso meses ${ }^{[2]}$.

Los requerimientos básicos de los sistemas vestibles descritos por Kumari, et al. [3], incluyen factores como la seguridad del paciente en todo momento, bajo consumo, diseño compacto, resistencia a factores ambientales, seguridad de la información y la estética del sistema. Recientemente, también se ha buscado integrar el Internet de las Cosas (IoT) a los sistemas vestibles, para así ofrecer sistemas personalizados capaces de transmitir la información, no sólo al paciente, sino también al personal médico ${ }^{[4-6]}$.

En 2010, Pantelopoulos y Bourbakis ${ }^{[7]}$, realizaron una revisión de los sistemas vestibles disponibles a la fecha. La mayor parte de los sistemas revisados adquieren más de una señal, es decir, son multiparamétricos. Las señales comúnmente adquiridas son el electrocardiograma (ECG), electromiograma (EMG), fotopletismograma (FPG), respiración, presión arterial, saturación de oxígeno y la temperatura corporal. Los sistemas vestibles también se han usado para detectar señales biomecánicas, como el movimiento de las extremidades, aplicado a pacientes con epilepsia mediante un prototipo montado en un guante ${ }^{[8]}$. Manimaraboopathy et al. ${ }^{[9]}$, desarrollaron un sistema que adquiere la fre- cuencia cardiaca, presión sanguínea y temperatura corporal, inclusive incorporaron un desfibrilador externo automatizado. También se han propuesto sistemas incorporados en camisas que detectan seis variables fisiológicas ${ }^{[10]}$, pulseras para monitorear la presión sanguínea, el ECG y/o FPG ${ }^{[11,12]}$, collares para el monitoreo a largo plazo de la actividad cardiaca ${ }^{[13]}$, sensores colocados en el oído para la detección de fuerzas de reacción contra el suelo ${ }^{[14]}$ o el FPG ${ }^{[15]}$ y anillos que estiman la frecuencia cardiaca y la temperatura corporal ${ }^{[16]}$, entre otros.

La ventaja de medir más de una variable fisiológica es que éstas se pueden correlacionar para estimar de forma indirecta otra variable. Un caso muy común es detectar el ECG y el FPG para estimar cambios de la presión arterial, sin usar un brazalete, mediante la medida del tiempo de transito de la onda de pulso (PTT) ${ }^{[17]}$. No obstante, el inconveniente de medir el FPG es que los diodos emisores de luz (LED) que se usan consumen mucha energía, lo cual no es una característica muy atractiva para un sistema portable. Para contrarrestar este problema, se han propuesto técnicas de modulación de la alimentación de los LEDs ${ }^{[18]}$, pero se requiere de una instrumentación más compleja y no tan compacta para extraer la información de interés.

En este trabajo se propone un sistema vestible capaz de detectar simultáneamente el ECG y la señal de flujo sanguíneo. Para adquirir el ECG se usan sólo dos electrodos secos y para adquirir el flujo sanguíneo se usa un sensor magnetorresistivo y un imán permanente. Por un lado, con esta propuesta no hay necesidad de usar LEDs y fotodetectores para detectar la actividad cardiaca en el lecho vascular, por lo que representa una técnica que requiere una instrumentación más compacta, de menor consumo de energía y más simple de implementar. Además, hasta donde sabemos, no hay evidencia de trabajos que hayan implementado esta técnica, en conjunto con el ECG, en un sistema vestible. Por otro lado, el uso de sólo dos electrodos 
para adquirir el ECG, simplifica mucho el diseño del bioamplificador. El objetivo de este trabajo es adquirir el ECG y la señal de flujo sanguíneo con una elevada relación señal a ruido (SNR) mediante un sistema que pueda llevarse como una prenda de vestir. Se deja para un trabajo a futuro la correlación de dichas señales, de tal forma que se pueda estimar indirectamente información adicional de la salud cardiovascular, como por ejemplo, la presión arterial.

\section{Sistema vestible}

\section{Diseño del brazalete}

El sistema vestible que se presenta en este trabajo tiene la forma de un brazalete, en el cual se incorporan dos electrodos secos, un sensor magnetorresistivo y un imán permanente de tamaño reducido. El diseño del brazalete dependerá de los electrodos secos que se van a escoger; básicamente el criterio de selección dependerá del material, del área de contacto y de la impedancia de los electrodos. Uno de los electrodos estará en contacto permanente con la muñeca donde el sujeto lleve puesto el brazalete. El segundo electrodo queda expuesto para que pueda hacer contacto con la otra muñeca y poder así adquirir la derivación I del ECG. En el caso del sensor magnetorresistivo, éste se debe colocar de tal forma que su posición sobre la muñeca coincida con la arteria radial del sujeto. El imán permanente se debe colocar a una distancia del sensor no mayor a $3 \mathrm{~cm}$, para no degradar la calidad de la señal de flujo sanguíneo ${ }^{[19]}$.

\section{Instrumentación}

\section{Adquisición del ECG mediante}

\section{dos electrodos secos}

Cuando se adquiere el ECG mediante dos electrodos, la contribución de las interferencias electromagnéticas depende del valor de la impedancia en modo común del amplificador al cual se conectan los electrodos. Esto se puede ver en el modelo propuesto por Spinelli et al. ${ }^{[20]}$ que describe la contribución de las interferencias electromagnéticas a la entrada del amplificador (para dos electrodos) de la siguiente manera:

$$
V_{\mathrm{iEMI}}=i_{\mathrm{P}} \frac{Z_{\mathrm{B}}}{Z_{\mathrm{C}}+2\left(Z_{\mathrm{B}}+Z_{\mathrm{ISO} O}\right)}\left(\Delta Z_{\mathrm{E}}+\frac{Z_{\mathrm{C}}}{\mathrm{CMRR}}\right),
$$

donde $i_{\mathrm{p}}$ es la corriente de desplazamiento proveniente de los $60 \mathrm{~Hz}$ y que circula por el sujeto; $Z_{\mathrm{B}}$ es la impedancia entre el sujeto y tierra; $Z_{\text {ISO }}$ es la impedancia de aislamiento entre la referencia del amplificador y tierra; $Z_{\mathrm{C}}$ es la impedancia en modo común del amplificador, $\Delta Z_{\mathrm{E}}\left(Z_{\mathrm{E} 1}-Z_{\mathrm{E} 2}\right)$ es la diferencia de impedancia de contacto entre el Electrodo 1 y el Electrodo 2; CMRR es la relación de rechazo en modo común del amplificador. De la Ecuación (1) se deduce que para reducir la contribución de las interferencias electromagnéticas, se debe tener valores elevados del CMRR, $Z_{\mathrm{B}}, Z_{\text {ISO }}$, y una reducida diferencia entre las impedancias de contacto de los electrodos 1 y 2 . No obstante, en lo que se refiere al valor de $Z_{\mathrm{C}}$, un valor nulo o infinito reducen la contribución de $V_{\text {iEMI }}$; la mejor estrategia, eso sí, dependerá del valor de $\Delta Z_{\mathrm{E}}{ }^{[20]}$.

En la Figura 1, se muestra el circuito utilizado para adquirir el ECG mediante dos electrodos secos. Todo el sistema está referenciado a $\mathrm{V}_{\mathrm{CC}} / 2$, ya que está pensado para ser alimentado con baterías. La etapa frontal consta de un amplificador completamente diferencial $\mathrm{y}$ es una modificación del circuito propuesto por Ramos et al. ${ }^{[21]}$, cuyas impedancias de entrada en modo diferencial $\left(Z_{\mathrm{D}}\right)$ y en modo común $\left(Z_{\mathrm{C}}\right)$ son:

$$
\begin{aligned}
& Z_{\mathrm{D}}=Z+Z^{\prime}+\frac{Z \prime Z}{R_{\mathrm{C}}} \\
& Z_{\mathrm{C}}=Z+R_{\mathrm{C}}+\frac{Z R_{\mathrm{C}}}{Z^{\prime}},
\end{aligned}
$$

donde

$$
\begin{gathered}
Z=\frac{1}{C_{2} s}+R_{1}+R_{2}+s R_{1} R_{2} C_{1} \\
Z^{\prime}=\frac{1}{C_{2} s}+R_{1}^{\prime}+R_{2}^{\prime}+s R_{1}^{\prime} R^{\prime}{ }_{2} C_{1}^{\prime}
\end{gathered}
$$




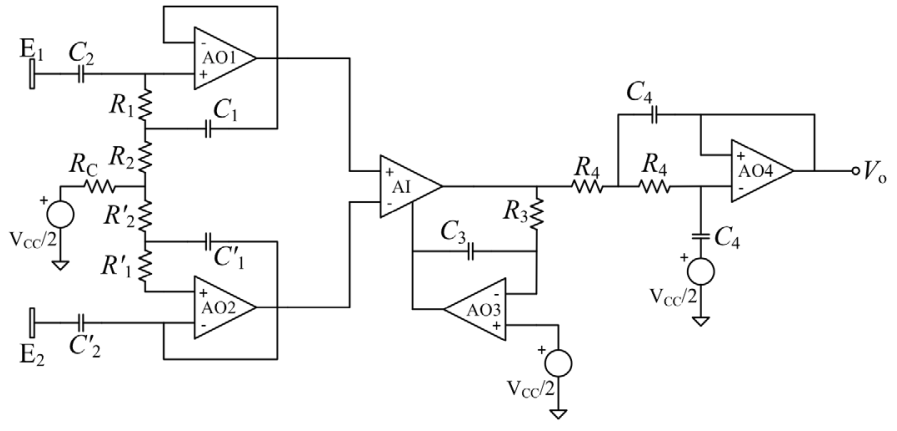

FIGURA 1. Circuito utilizado para adquirir el ECG mediante 2 electrodos secos.

De las Ecuaciones (2)-(5), se puede ver que es posible fijar los valores de $Z_{\mathrm{D}}$ y $Z_{\mathrm{C}}$ eligiendo los valores de los componentes pasivos (resistencias y condensadores) de la etapa frontal. Por tanto, es fácil ajustar el valor de $Z_{\mathrm{C}}$ una vez que se sepa cuál estrategia (elevada o baja $Z_{\mathrm{C}}$ ) se debe usar para reducir $V_{\text {iEMI }}$.

La salida diferencial de la etapa frontal se conecta a las entradas de un amplificador de instrumentación, el cual tiene un circuito supresor de tensiones en c.c. para eliminar los errores de cero a la salida. Finalmente, a la salida del sistema se conecta un filtro activo pasobajo de segundo orden para limitar el ancho de banda del sistema al margen de frecuencias del ECG. Debido a que este sistema está pensado para fines de monitorización (no de diagnóstico), es suficiente un ancho de banda de $40 \mathrm{~Hz}{ }^{[22]}$. Para implementar el circuito de la Figura 1, se utilizó el amplificador de instrumentación (AI) LT1167, de Linear Technology el cual tiene un consumo de corriente $900 \mu \mathrm{A}$; para los cuatro amplificadores operacionales (AO), se utilizó el LMP7704 de Texas Instruments, donde cada amplificador consume $715 \mu \mathrm{A}$.

\section{Detección del flujo sanguíneo}

Para detectar el flujo sanguíneo se utilizó el método propuesto por Phua ${ }^{[23]}$. Cuando un campo magnético uniforme se aplica sobre la piel, cerca de arterias de gran tamaño, el flujo pulsátil de la sangre arterial produce una distorsión de dicho campo; esto se debe a que la sangre se comporta como un fluido con propiedades magnéticas afectadas por su estado de oxigenación. Si el campo magnético generado (por un imán permanente, por ejemplo) abarca a un sensor magnético, éste último detecta la distorsión que produce el flujo de sangre arterial en dicho campo. En este trabajo se utilizó un sensor magnetorresistivo que tiene cuatro magnetorresistencias conectadas en una configuración de puente de Wheatstone (completo). El campo magnético proviene de un imán de neodimio de $10 \mathrm{x}$ $10 \times 3 \mathrm{~mm}$. La Figura 2 muestra el circuito utilizado para procesar la señal del sensor magnetorresistivo. El circuito consta de un AI acoplado en alterna mediante un filtro paso-alto completamente diferencial para garantizar un CMRR elevado. De esta forma se eliminan los errores de cero provenientes del sensor, y sólo se amplificaría la señal alterna de interés. El amplificador operacional está configurado de tal forma que se eliminen los errores de cero a la salida del AI. Dichos errores de cero son productos de las corrientes de polarización y de los errores de cero del propio AI. Finalmente, un filtro paso-bajo pasivo de primer orden limita el ancho de banda del sistema a $10 \mathrm{~Hz}$, suficiente para adaptarse al margen de frecuencias de la señal de flujo sanguíneo ${ }^{[24]}$. Debido a que el sistema está pensado para alimentarse con baterías, se utilizó el AI AD627 de Analog Devices y el AO TLV2454 de Texas Instruments, los cuales consumen $60 \mu \mathrm{A}$ y 40 $\mu \mathrm{A}$, respetivamente.

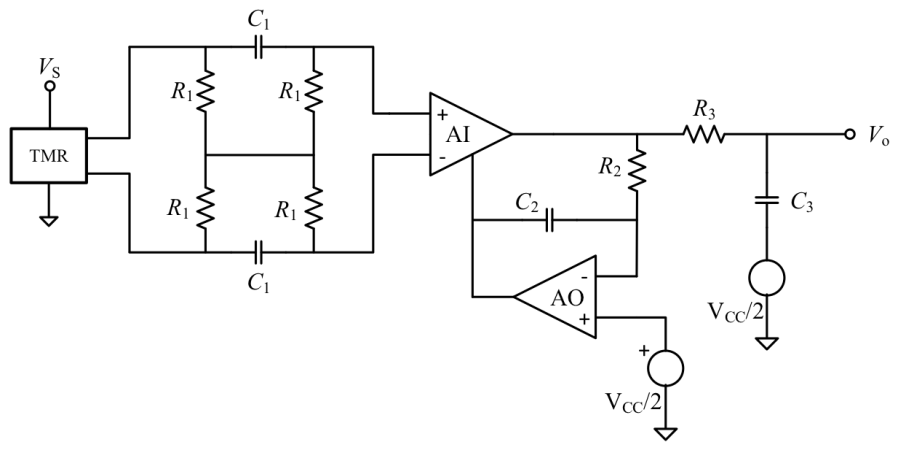

FIGURA 2. Circuito utilizado para adquirir la señal de flujo sanguíneo mediante un sensor magnetorresistivo. 


\section{METODOLOGÍA}

\section{Selección y caracterización de los electrodos secos}

Para seleccionar los electrodos secos hay que considerar el material a utilizar, ya que de esto depende el potencial de media celda, así como el tiempo de estabilización de la interfaz electrodo-piel ${ }^{[25]}$. Con base en estos criterios, los electrodos de $\mathrm{Ag}-\mathrm{AgCl}$ resultan ser la mejor opción pues, al ser no polarizables (resistivos), permiten medir desde c.c. Además, presentan una baja tensión de polarización (220 mV), una baja deriva de línea base $(0.13 \mathrm{mV})$ a $25^{\circ} \mathrm{C}^{[26]} \mathrm{y}$ un periodo relativamente corto de estabilización ${ }^{[27]}$. También, hay que considerar el tamaño y forma del electrodo, lo cual influye en gran medida en la impedancia de contacto y en el ruido de la interfaz electrodo-piel. Al aumentar el área de contacto, tanto el ruido de la interfaz como la impedancia de contacto, disminuyen ${ }^{[27]}$.

Para seleccionar los electrodos que se van a utilizar en el sistema propuesto en este trabajo, se caracterizó la impedancia de la interfaz electrodo-piel de distintos electrodos (Tabla 1). El material de los distintos electrodos caracterizados es de Ag-AgCl. Lo que se busca es comparar la impedancia de la interfaz electrodo-piel utilizando electrodos de distintas marcas y tamaños. Los pares de electrodos del 1 al 4 son electrodos originalmente húmedos, pero se les removió el gel electrolítico con el fin de utilizarlos como electrodos secos. El par de electrodos número 5 son electrodos originalmente secos, pero con una mayor área de contacto. El par de electrodos número 6 son electrodos húmedos y fueron caracterizados para compararlos con los secos.

La caracterización de los electrodos consistió en medir la impedancia de contacto en un margen de frecuencias determinado. Para ello, el par de electrodos a caracterizar se colocó sobre la parte interna del antebrazo de un sujeto de prueba, separados por una distancia de $2 \mathrm{~cm}$, un electrodo del otro, con la finalidad de minimizar la
TABLA 1. Electrodos caracterizados.

\begin{tabular}{cccc}
\hline \hline $\begin{array}{c}\text { Par de } \\
\text { electrodo }\end{array}$ & $\begin{array}{c}\text { Tipo de } \\
\text { contacto }\end{array}$ & Marca & $\begin{array}{c}\text { Área de } \\
\text { contacto de } \\
\text { cada } \\
\text { electrodo } \\
\left.\mathbf{( c m}^{\mathbf{2}}\right)\end{array}$ \\
\hline 1 & Seco & Cathay & 0.7853 \\
2 & Seco & AMBIDERM & 0.7853 \\
3 & Seco & Kendall Q- & 0.7853 \\
4 & Reco & 3M 2228 & 0.7853 \\
5 & Seco & Kenz & 10.5 \\
6 & Húmedo & $3 \mathrm{M} 2228$ & 0.7853 \\
\hline \hline
\end{tabular}

contribución de la impedancia del tejido. Para medir la impedancia de contacto se utilizó un analizador de impedancia E4990A de Keysight, dispuesto para realizar una medida a 4 hilos mediante un adaptador (modelo 16089D) que sirve para realizar este tipo de medidas. El instrumento se configuró para trabajar en un margen de frecuencias de $20 \mathrm{~Hz}$ hasta $100 \mathrm{kHz}$. Se usó este margen de frecuencias ya que representa el límite de frecuencias que tiene el instrumento al realizar medidas con el adaptador utilizado. Esta prueba se realizó para cada par de electrodos de la Tabla 1 en el mismo sujeto de prueba. Es importante señalar que con este método no es posible medir la impedancia individual de cada electrodo, sino la de ambos.

Cuando se usan electrodos secos, la impedancia de contacto depende de las condiciones de la piel. Por tanto, si se repitiera el proceso en diferentes sujetos, se obtendrían impedancias distintas en cada caso. En este trabajo sólo se realizó la prueba en un sólo sujeto para determinar cuál de los pares caracterizados presentaba la menor impedancia, lo cual es una valoración que se puede realizar con un solo sujeto.

\section{Caracterización estática y dinámica del sensor magnetorresistivo}

En la Figura 3 se muestra la configuración utilizada para caracterizar estáticamente al sensor magnetorresistivo. En este trabajo se utilizó un sensor tipo Túnel, 
TMR2505 de Multi Dimension. Con el sistema mostrado en la Figura 3 se midió la tensión de salida del sensor para distintos valores de un campo magnético constante. Para ello, se alimentó a un inductor de 24 $\mu \mathrm{H}$ con una tensión c.c. desde $2 \mathrm{mV}$ hasta $30 \mathrm{mV}$. Esto se hizo para generar un campo magnético no mayor a $190 \mu \mathrm{T}$, punto en el cual se saturaba el magnetómetro Bartington, Mag-01H que se usó para medir el campo magnético generado por el inductor. La tensión de salida diferencial del sensor se midió con un multímetro digital de 6.5 dígitos, Keysight 34461A configurado para medir tensiones c.c. Debido a que se trata de un sensor modulador, la caracterización estática se realizó para cuando el sensor se alimentó a una tensión c.c. de $1 \mathrm{~V}$ y para una tensión c.c. de $5 \mathrm{~V}$, y así valorar cómo la tensión de alimentación afecta a la sensibilidad del sensor.

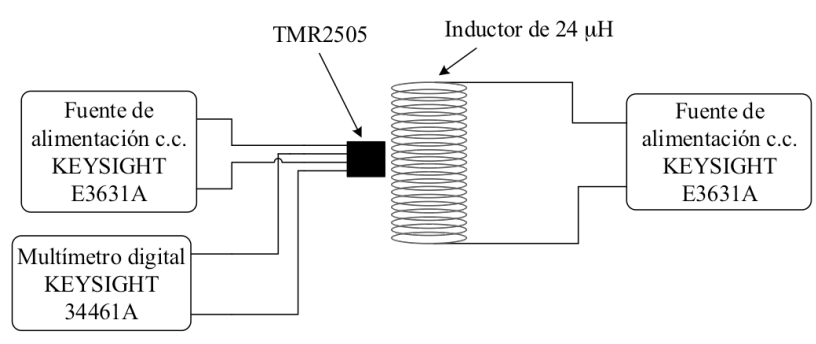

FIGURA 3. Configuración utilizada para caracterizar estáticamente al sensor TMR2505.

En la Figura 4 se muestra la configuración utilizada para caracterizar dinámicamente al sensor TMR2505. En esta oportunidad, el inductor se conectó a un generador de forma de ondas 33500B de Keysight, cuya salida analógica se configuró para obtener una tensión de $10 \mathrm{~V}$ de pico a pico en un margen de frecuencias entre $0.1 \mathrm{~Hz}$ y $100 \mathrm{~Hz}$. El sensor se alimentó con una tensión c.c. de $5 \mathrm{~V}$ y su salida diferencial se conectó a un AI, AD620 de Analog Devices, con una ganancia de 10. Para los distintos valores de frecuencia, se midió la tensión pico a pico a la salida del AI con un osciloscopio Keysight DSOX2014A. Debido a que el sensor magnetorresistivo se emplea para medir cambios en el campo magnético producidos por el paso de la sangre arterial, lo que se busca con este procedimiento es valorar la respuesta que tiene el sensor TMR2505 para medir un campo magnético alterno $\boldsymbol{B}(t)$ a diferentes frecuencias.

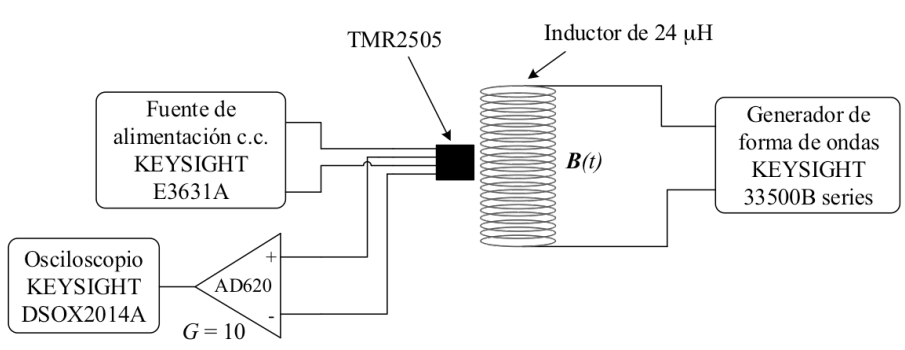

\section{FIGURA 4. Configuración utilizada para \\ la caracterización dinámica del sensor TMR2505.}

\section{Caracterización de la instrumentación}

A cada uno de los circuitos implementados en este trabajo (Figura 1 y Figura 2) se le estimaron experimentalmente distintas características eléctricas como: ganancia total, CMRR, errores de cero, respuesta en frecuencia, ruido electrónico y consumo de corriente.

\section{Protocolo de medida}

Se adquirieron las señales de ECG y flujo sanguíneo de 10 sujetos de prueba (4 hombres y 6 mujeres) de distintas edades, pesos y alturas (media $\pm \sigma$ : edad $=(22$ $\pm 1)$ años; peso = $(75 \pm 24) \mathrm{kg}$; altura $=(1.69 \pm 0.13) \mathrm{m})$. A cada sujeto se le pidió permanecer sentado y relajado con sus brazos extendidos sobre una mesa mientras usaba el brazalete. El tiempo de cada medida fue de 5 s. Simultáneamente se detectó la derivación I del ECG mediante el sistema AD8232, de Analog Devices usando tres electrodos húmedos. Las medidas obtenidas con este sistema se consideraron como de referencia para compararlas con las medidas obtenidas con el sistema propuesto en este trabajo. Las señales provenientes de los sistemas desarrollados, así como el ECG de referencia, fueron adquiridas simultáneamente utilizando una tarjeta de adquisición de datos NI USB- 6341 de National Instruments, de 16 bits, configurada para realizar la adquisición mediante tres entradas analógicas a una frecuencia de muestreo de $1 \mathrm{kHz}$ cada una. 


\section{RESULTADOS Y DISCUSIÓN}

En la Figura 5 se muestra el módulo de la impedancia de contacto de los seis pares de electrodos caracterizados. Se puede ver que los electrodos que presentaron una menor impedancia de contacto fueron los electrodos de mayor área (\#5 de la Tabla 1). Independientemente de si los electrodos eran secos o húmedos, todos presentaron un descenso de la impedancia a medida que aumentaba la frecuencia; esto se debe gracias a la componente capacitiva que tiene la interfaz electrodo-piel (para los electrodos secos) y la interfaz electrodo-electrolito-piel (para los electrodos húmedos). A partir de estos datos, se decidió trabajar con el par de electrodos \#5, el cual presentó una impedancia inferior a $40 \mathrm{k} \Omega$ (unos $20 \mathrm{k} \Omega$ cada electrodo, aproximadamente). Con esta impedancia, es posible reducir considerablemente los errores por efectos de carga cuando los electrodos se conecten a la etapa frontal del amplificador de la Figura 1.

Debido a que el método utilizado para determinar la impedancia de los electrodos no es capaz de estimar la impedancia individual de cada electrodo, es difícil saber el valor de $\Delta Z_{\mathrm{E}}$ para así valorar cuál es la mejor estrategia (incrementar o reducir el valor de $Z_{\mathrm{C}}$ ) para

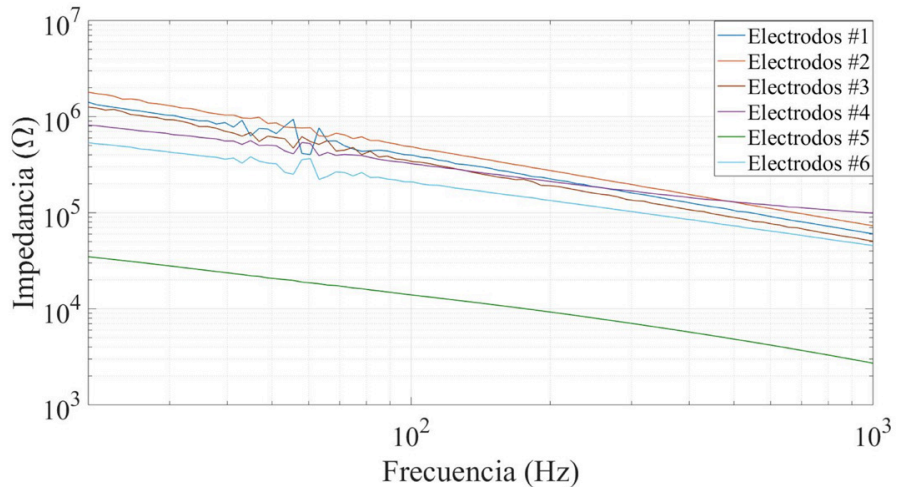

FIGURA 5. Módulo de la impedancia de contacto de cada uno de los 6 pares de electrodos caracterizados.

minimizar el valor de $V_{\text {iemI }}$. Por ese motivo, antes de implementar el circuito de la Figura 1, se realizó una prueba propuesta por Spinelli y Mayosky ${ }^{[20]}$, donde es posible realizar la medida del ECG para un valor bajo $(100 \Omega)$ y un valor elevado (700 $\mathrm{M} \Omega$ ) de $Z_{\mathrm{C}}$ y observar la contribución de la interferencia de línea para cada caso. El resultado de esa prueba se muestra en la Figura 6 cuando el ECG se adquirió con el par de electrodos \#5. Se puede ver cómo la contribución de la interferencia de línea se reduce para un valor elevado de $Z_{C}$. Por tanto, la etapa frontal del circuito de la Figura 1 se diseñó para que tuviera una $Z_{\mathrm{C}}=3 \mathrm{G} \Omega$.

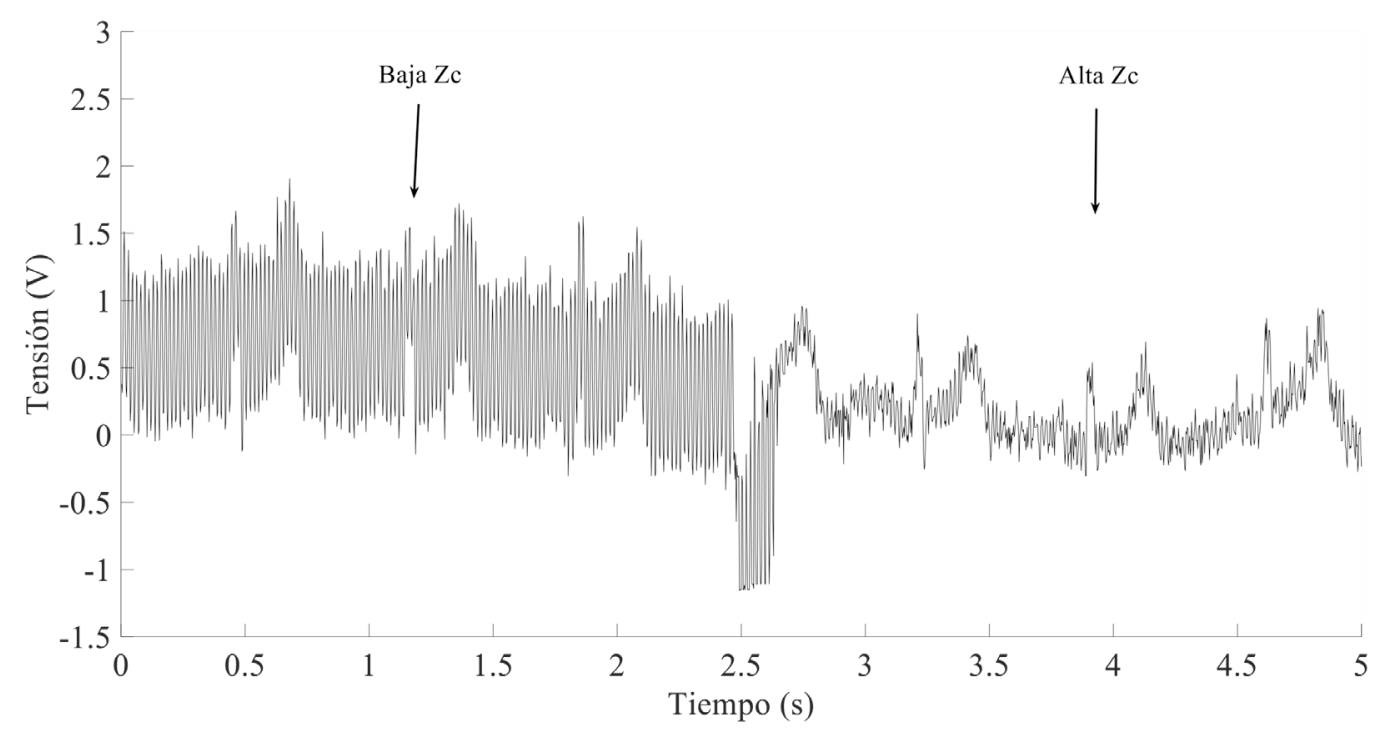

FIGURA 6. Efecto de una baja y una elevada $Z_{c}$ en la contribución de la interferencia de línea en el ECG adquirido mediante el par de electrodos \#5. 
En la Figura 7 se muestra la curva de sensibilidad estática del sensor TMR2505 para una tensión de alimentación de $1 \mathrm{~V}$ y de $5 \mathrm{~V}$. Al aumentar la tensión de alimentación, tanto la sensibilidad como el error de cero, aumentan. Debido a que la perturbación que produce la sangre al campo magnético que detecta el sensor es muy pequeña, se decidió alimentar el sensor con una tensión de $5 \mathrm{~V}$, de esa forma, no habría necesidad de implementar un amplificador con una ganancia tan elevada, y así reducir la contribución de ruido electrónico y errores de cero a la salida del sistema.

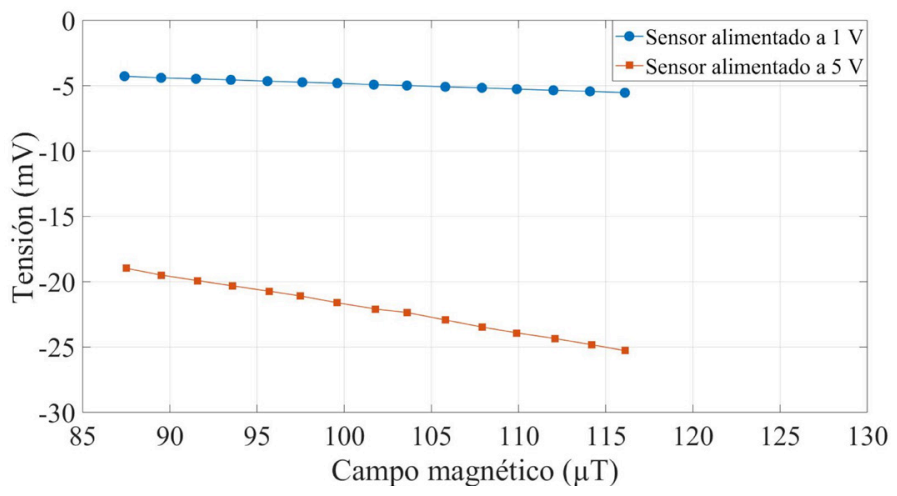

FIGURA 7. Sensibilidad estática del sensor TMR2505 para una tensión de alimentación de $1 \mathrm{~V}$ y de $5 \mathrm{~V}$.

La Figura 8 muestra la respuesta del sensor TMR2505 ante un campo magnético alterno entre $0.1 \mathrm{~Hz}$ y $100 \mathrm{~Hz}$. Se observa como el sensor tiene una respuesta plana en dicho margen de frecuencias. Sabiendo que la componente principal de frecuencia de la señal de flujo san-

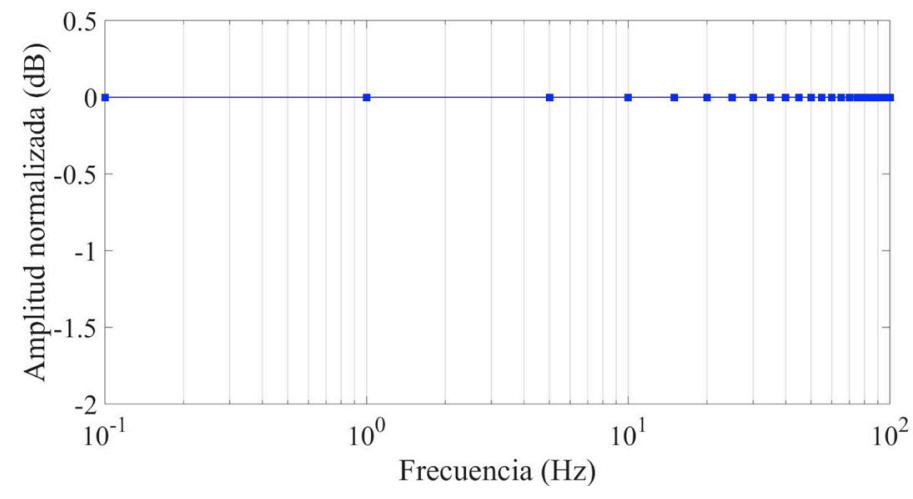

FIGURA 8. Respuesta dinámica del sensor TMR2505 ante un campo magnético alterno. guíneo está en el orden de los $10 \mathrm{~Hz}^{\text {[24] }}$, es evidente que el sensor magnetorresistivo utilizado tiene una respuesta dinámica suficiente para detectar dicha señal.

Una vez caracterizados los electrodos y el sensor magnetorresistivo, se procedió a caracterizar cada uno de los circuitos. Ambos circuitos se alimentaron con una tensión c.c. regulada de $5 \mathrm{~V}$ mediante una pila de 9 V. El circuito de la Figura 1 presentó un CMRR superior $88 \mathrm{~dB}$ a una frecuencia de $60 \mathrm{~Hz}$. El ancho de banda se configuró para tener una frecuencia inferior de $0.1 \mathrm{~Hz} \mathrm{y}$ una frecuencia superior de $40 \mathrm{~Hz}$. La ganancia total del circuito fue de unos $60 \mathrm{~dB}$, aproximadamente. Se obtuvo una tensión de ruido de pico a pico de $490 \mu \mathrm{V}$ referidos a la salida del circuito y un error de cero de 435 $\mu \mathrm{V}$. En lo que respecta al circuito de la Figura 2, se obtuvo una ganancia superior a $49 \mathrm{~dB}$ y un CMRR de unos $87 \mathrm{~dB}$ a la frecuencia de $60 \mathrm{~Hz}$. El ancho de banda del circuito quedó limitado entre $0.1 \mathrm{~Hz}$ y $10 \mathrm{~Hz}$. La tensión de ruido obtenido a la salida fue de $415 \mu \mathrm{V}$ de pico a pico con un error de cero de $357 \mu \mathrm{V}$. Cuando ambos sistemas trabajaban conjuntamente, se obtuvo un consumo de corriente de $10.44 \mathrm{~mA}$. Por tanto, usando una pila de $9 \mathrm{~V}$ con una capacidad de $170 \mathrm{mAh}$, el sistema tendría una autonomía de 16 horas, aproximadamente, realizando medidas de forma ininterrumpida.

En la Figura 9 se muestra el diseño del sistema vestible desarrollado en este trabajo. En la Figura 9a, se observa el Electrodo 1, el sensor TMR2505 y el imán permanente, los cuales hacen contacto con la piel del sujeto, quedando expuesto el Electrodo 2 (Figura 9b). En lo que respecta al sensor TMR2505, hay que cuidar que éste se coloque justo encima de la arteria radial, de lo contrario, no se obtendría la señal de flujo sanguíneo. En la Figura 9c se muestra cómo la otra muñeca hace contacto con el Electrodo 2, de esta forma, se obtiene la derivación I del ECG. Para evitar que los dos electrodos se desplacen de lugar, éstos se fijaron a una base diseñada en SolidWorks 2017 y se construyeron mediante impresión 3D usando ácido poliláctico (PLA). 


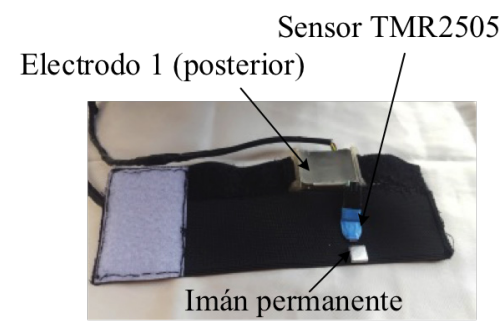

a)

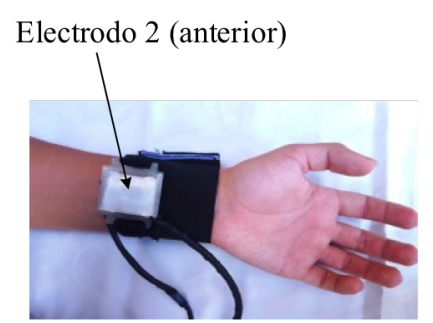

b)

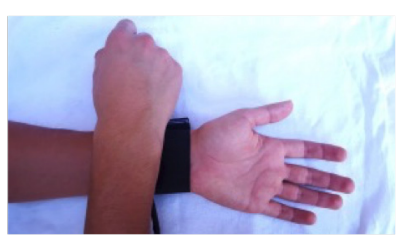

c)

FIGURA 9. a) Diseño final del brazalete donde se muestra uno de los electrodos (electrodo 1, posterior) el sensor TMR 2505 y el imán permanente. b) El brazalete colocado sobre una de las muñecas (electrodo 2, anterior). c) Posición de las manos para adquirir el ECG y el flujo sanguíneo.

Con el sistema vestible de la Figura 9 fue posible registrar simultáneamente el ECG y el flujo sanguíneo mediante dos electrodos secos y un sensor magnetorresistivo dispuestos en la muñeca de un sujeto (Figura 10). Las dos señales presentan una SNR superior a 41 dB. En el caso de la señal de flujo sanguíneo, la calidad de la señal depende de los siguientes factores: la posición del sensor sobre la arteria radial; la distancia entre el imán permanente y el sensor y de la presión que ejerce el sensor sobre la piel. En el caso del ECG, la calidad de la señal depende de las condiciones de la interfaz electrodo-piel, lo cual varía de sujeto a sujeto. En la Figura 11 se presenta el mejor y el peor caso del ECG registrado entre los 10 voluntarios. En el mejor caso, se obtuvo un ECG con una mínima contribución de ruido electrónico y de interferencias de línea; se aprecian las ondas $\mathrm{P}, \mathrm{Q}, \mathrm{R}, \mathrm{S}, \mathrm{T}$ y una línea isoeléctrica estable, siempre y cuando el sujeto no se mueva mientras se realiza la medida ( $5 \mathrm{~s}$ ). En el peor caso, el ECG posee una considerable contribución de ruido, el cual, se prevé, proviene de la interfaz electrodo-piel del sujeto de prueba. En este caso, sólo es posible distinguir la onda R, lo cual permite estimar la frecuencia cardiaca; también se distingue la onda T, aunque está deformada por el ruido electrónico. En el ECG registrado en los 10 sujetos, no hay contribución apreciable de interferencia de línea, esto gracias a que el circuito obtuvo un CMRR superior a $88 \mathrm{~dB}$ a una frecuencia de $60 \mathrm{~Hz}$.
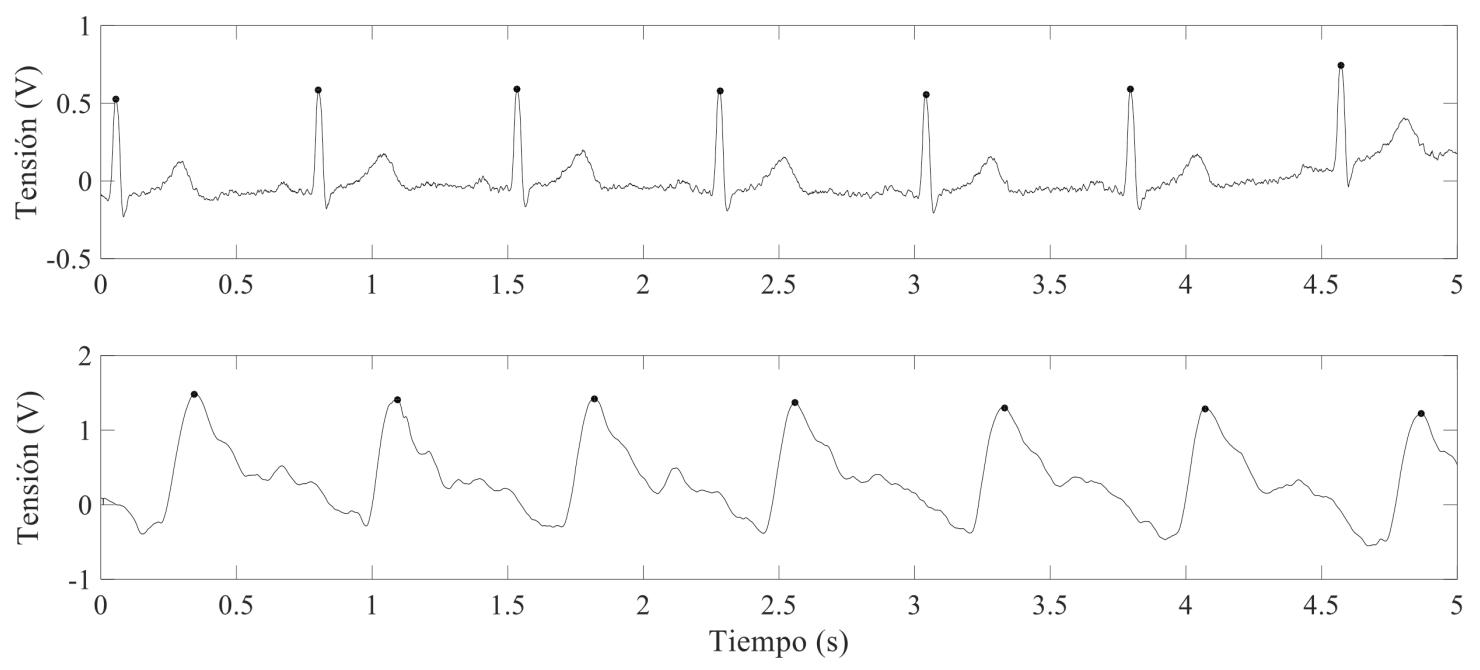

FIGURA 10. Señales de ECG (registro superior) y de flujo sanguíneo (registro inferior) obtenidas con el sistema propuesto. Las señales se registraron simultáneamente. 

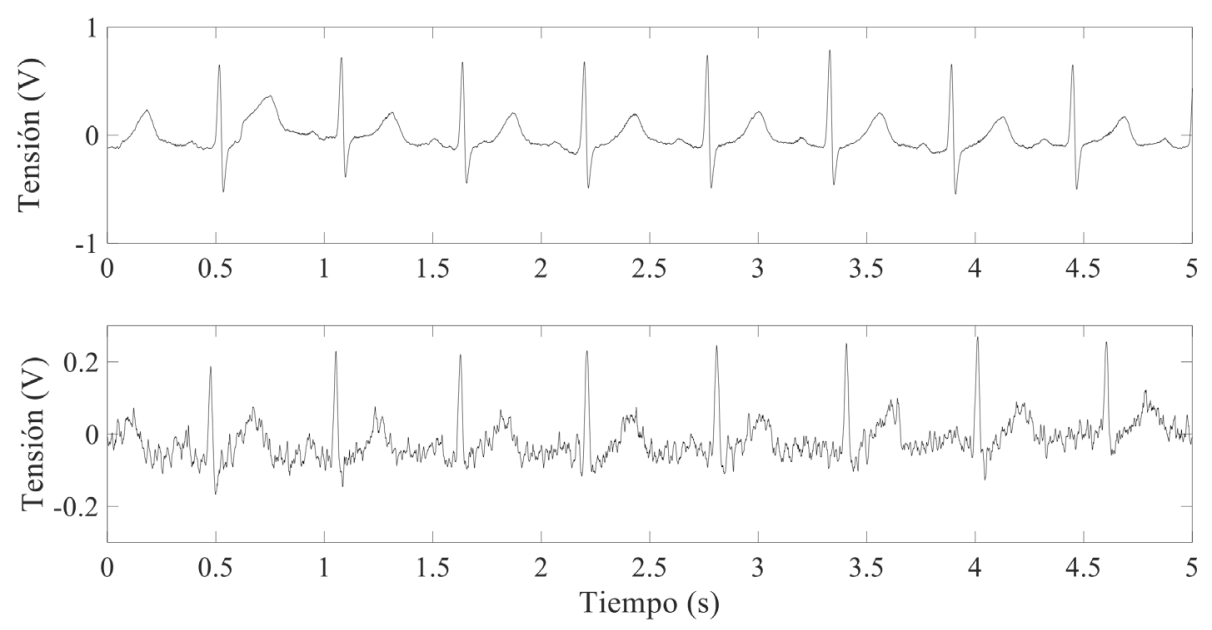

FIGURA 11. ECG obtenido mediante dos electrodos dispuestos en el sistema vestible. Mejor caso (registro superior) y peor caso (registro inferior) obtenido entre los 10 sujetos de prueba.

En la Figura 10, al comparar el ECG con la señal de flujo sanguíneo, se observa que existe un retardo entre el complejo QRS del ECG y el pico máximo de la señal de flujo sanguíneo. Dicho retardo se puede usar para estimar, por ejemplo, el PTT, el cual tiene un gran potencial en aplicaciones clínicas relacionadas con la valoración de la salud cardiovascular ${ }^{[17]}$.

Finalmente, para validar estadísticamente las medidas que se realizaron con el sistema vestible, se estimó el intervalo RR (latido a latido) de la señal de ECG adquirida con 2 electrodos secos (RR2e) y de la señal de flujo sanguíneo (RRfs). Estos valores se compara- ron con el intervalo RR del ECG adquirido con el sistema de referencia (RR3e). Para estimar el intervalo RR se detectaron los picos de cada una de las señales mediante un algoritmo sencillo implementado en MATLAB ${ }^{\circledR}$. Los resultados se observan en las gráficas Bland-Altman que se muestran en las Figuras 12 y 13, las cuales presentan las medidas latido a latido de los 10 voluntarios. En ambas gráficas se estimó la desviación estándar (SD) con un intervalo de confianza de 95 \% (2SD) obteniendo el peor caso (43.6 ms) cuando se estimó el intervalo RR en la señal de flujo sanguíneo, lo cual se debe en que en algunos de los voluntarios fue difícil localizar la arteria radial para posicionar el

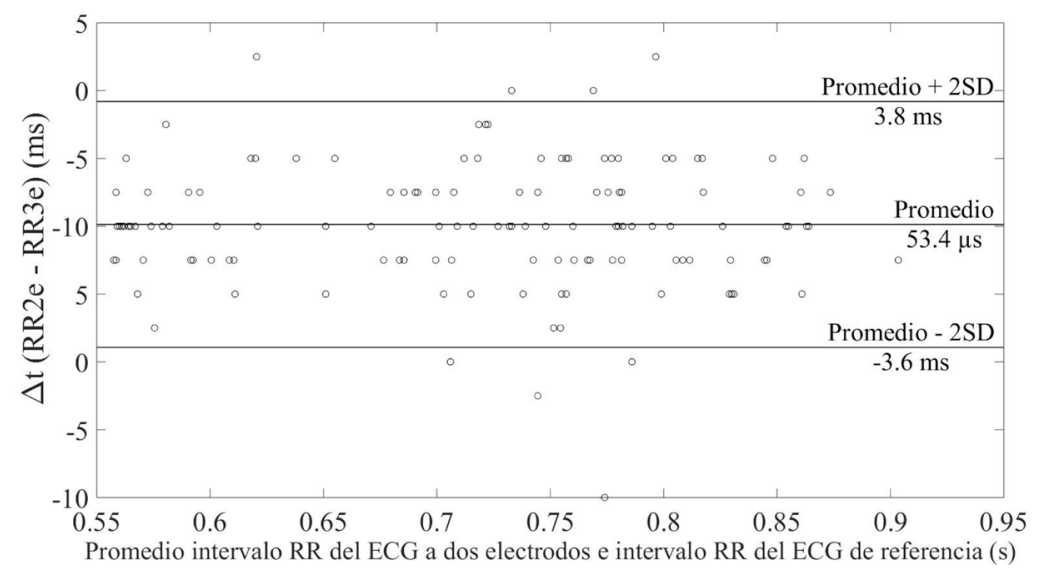

FIGURA 12. Gráfica Bland-Altman donde se comparan los intervalos RR (latido a latido) del ECG obtenido con el sistema propuesto y el ECG obtenido con el sistema de referencia. 


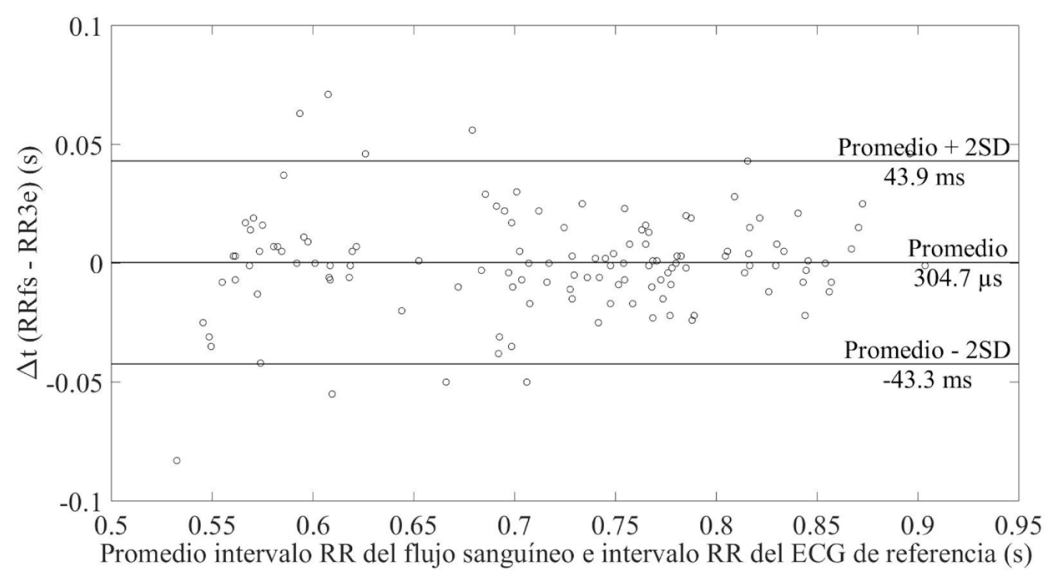

FIGURA 13. Gráfica Bland-Altman donde se comparan los intervalos RR (latido a latido) de la señal de flujo sanguíneo obtenido con el sistema propuesto y el ECG obtenido con el sistema de referencia.

sensor TMR2505. Aun así, se demuestra que existe una excelente coincidencia entre las señales obtenidas con el sistema propuesto y el ECG de referencia.

\section{CONCLUSIONES}

En este trabajo se ha demostrado la posibilidad de adquirir simultáneamente dos señales cardiacas mediante un sistema vestible tipo brazalete. El ECG se adquirió mediante dos electrodos secos, y aun así, la señal detectada no presentó evidencia de la contribución de interferencia de $60 \mathrm{~Hz}$. Se detectó la actividad cardiaca en la muñeca del usuario mediante un sensor magnetorresistivo, sin necesidad de usar sensores ópticos, los cuales consumen mucha más energía. La calidad de las señales superó los 41 dB de SNR, siempre y cuando el sujeto no se moviera durante los $5 \mathrm{~s}$ que duraba la medida. En este trabajo sólo los sensores se incorporaron al brazalete, no la instrumentación; ésta se implementó con dispositivos de bajo consumo, per- mitiendo alimentar el sistema a pilas. En caso de que la capacidad de la pila sea de $170 \mathrm{mAh}$, el sistema puede trabajar ininterrumpidamente durante 16 horas. Obviamente, este tiempo puede aumentarse gestionando el consumo de energía. Las medidas se validaron estadísticamente demostrando una excelente coincidencia entre el intervalo RR (latido a latido) de las señales adquiridas con el brazalete y el intervalo RR (latido a latido) del ECG de referencia. El sistema presentado en este trabajo es una alternativa a los sistemas vestibles propuestos hasta ahora, y que permite estimar indirectamente otras variables fisiológicas, como como cambios de la presión arterial o la variabilidad de la frecuencia cardiaca en ambientes no hospitalarios.

\section{RECONOCIMIENTOS}

El presente estudio fue parcialmente financiado por la Universidad Autónoma de Ciudad Juárez y el Programa para el Desarrollo Profesional Docente (PRODEP). 


\section{REFERENCIAS}

[1] Bhelkar V, Shedge D. Different types of wearable sensors and health monitoring systems: A survey. 2nd IEEE International Conference on Applied and Theoretical Computing and Communication Technology, 2016. DOI: 10.1109/ ICATCCT.2016.7911963

[2] Chan M, Estève D, Fourniols J-Y, Escriba C, Campo E. Smart wearable systems: Current status and future challenges. Artificial intelligence in medicine. 2012;56(3):137-56. DOI: https://doi. org/10.1016/j.artmed.2012.09.003

[3] Kumari P, Mathew L, Syal P. Increasing trend of wearables and multimodal interface for human activity monitoring: A review. Biosensors and Bioelectronics. 2017;90:298-307. DOI: https://doi. org/10.1016/j.bios.2016.12.001

[4] Metcalf D, Milliard ST, Gomez M, Schwartz M. Wearables and the internet of things for health: Wearable, interconnected devices promise more efficient and comprehensive health care. IEEE pulse. 2016;7(5):35-9. DOI: 10.1109/MPUL.2016.2592260

[5] Anzanpour A, Azimi I, Götzinger M, Rahmani AM, TaheriNejad N, Liljeberg P, et al. Self-awareness in remote health monitoring systems using wearable electronics. Proceedings of the Conference on Design, Automation \& Test in Europe; 2017.

[6] Qi J, Yang P, Min G, Amft O, Dong F, Xu L. Advanced internet of things for personalised healthcare systems: A survey. Pervasive and Mobile Computing. 2017;41:132-49.DOl: https://doi. org/10.1016/j.pmcj.2017.06.018

[7] Pantelopoulos A, Bourbakis NG. A survey on wearable sensor-based systems for health monitoring and prognosis. IEEE Transactions on Systems, Man, and Cybernetics, Part C (Applications and Reviews). 2010;40(1):1-12. DOI: 10.1109/TSMCC.2009.2032660

[8] Ramirez-Alaminos JM, Sendra S, Lloret J, Navarro-Ortiz J. Lowcost wearable bluetooth sensor for epileptic episodes detection. IEEE International Conference on Communications 2017. DOI: 10.1109/ICC.2017.7997413

[9] Manimaraboopathy M, Vijayalakshmi S, Hemavathy D, Priya A. A Wearable Multiparameter Medical Monitoring and Alert System with First Aid. International Journal on Smart Sensing \& Intelligent Systems. 2017;10.

[10] Pandian P, Mohanavelu K, Safeer K, Kotresh T, Shakunthala D, Gopal P, et al. Smart Vest: Wearable multi-parameter remote physiological monitoring system. Medical engineering and physics. 2008;30(4):466-77. DOI: https://doi.org/10.1016/i.medengphy.2007.05.014

[11] Poon CC, Wong YM, Zhang Y-t. M-health: the development of cuffless and wearable blood pressure meters for use in body sensor networks. IEEE Life Science Systems and Applications Workshop, 2006.DOI: 10.1109/LSSA.2006.250377

[12] Thomas SS, Nathan V, Zong C, Aroul P, Philipose L, Soundarapandian K, et al. Demonstration abstract: BioWatch-A wrist watch based physiological signal acquisition system. Proceedings of the 13th International Symposium on Information Processing in Sensor Networks, 2014. DOI: 10.1109/ IPSN.2014.6846797
[13] Penders J, van de Molengraft J, Altini M, Yazicioglu F, Van Hoof C. A low-power wireless ECG necklace for reliable cardiac activity monitoring on-the-move. Proceedings of the International Conference of the IEEE Engineering in Medicine and Biology Society, 2011.

[14] Lo B, Pansiot J, Yang G-Z. Bayesian analysis of sub-plantar ground reaction force with BSN. IEEE Sixth International Workshop on Wearable and Implantable Body Sensor Networks, 2009. DOI: $10.1109 / B S N .2009 .38$

[15] Mouradian V, Poghosyan A, Hovhannisyan L. Continuous wearable health monitoring using novel PPG optical sensor and device. IEEE 10th International Conference on Wireless and Mobile Computing, Networking and Communications, 2014. DOl: 10.1109/ WiMOB.2014.6962159

[16] Wu Y-C, Chang C-S, Sawaguchi Y, Yu W-C, Chen M-J, Lin J-Y, et al. A mobile-phone-based health management system. Health Management-Different Approaches and Solutions: InTech; 2011.

[17] Smith RP, Argod J, Pépin J-L, Lévy PA. Pulse transit time: an appraisal of potential clinical applications. Thorax. 1999;54(5):452-7. DOI: http://dx.doi.org/10.1136/thx.54.5.452

[18] Asada HH, Shaltis P, Reisner A, Rhee S, Hutchinson RC. Mobile monitoring with wearable photoplethysmographic biosensors. IEEE engineering in medicine and biology magazine. 2003;22(3):28-40. DOI: 10.1109/MEMB.2003.1213624

[19] Phua CT, Lissorgues G. Modeling of pulsatile blood flow in a weak magnetic field. World Academy of Science, Engineering and Technology. 2009;54.

[20] Spinelli EM, Mayosky MA. Two-electrode biopotential measurements: power line interference analysis. IEEE Transactions on biomedical engineering. 2005;52(8):1436-42.

[21] Ramos J, Pallas-Areny R, Tresanchez M. Multichannel front-end for low level instrumentation signals. Measurement. 1999;25(1):41-6. DOI: https://doi.org/10.1016/S0263-2241(98)00065-7

[22] Instrumentation AftAoM. Cardiac monitors, heart rate meters, and alarms. American National Standard (ANSI/AAMI EC13: 2002) Arlington, VA. 2002:1-87.

[23] Phua CT. Novel method of blood pulse and flow measurement using the disturbance created by blood flowing through a localized magnetic field: Université Paris-Est; 2012.

[24] Webster J. Medical instrumentation: application and design: John Wiley \& Sons; 2009.

[25] Searle A, Kirkup L. A direct comparison of wet, dry and insulating bioelectric recording electrodes. Physiological Measurement. 2000;21(2):271.

[26] Xu J, Mitra S, Van Hoof C, Yazicioglu RF, Makinwa KA. Active electrodes for wearable EEG acquisition: Review and electronics design methodology. IEEE reviews in biomedical engineering. 2017;10:187-98. DOI: 10.1109/RBME.2017.2656388

[27] Huigen E, Peper A, Grimbergen C. Investigation into the origin of the noise of surface electrodes. Medical and biological engineering and computing. 2002;40(3):332-8. 